\title{
Context-based Estimation of Driver Intent at Road Intersections
}

\author{
Stéphanie Lefèvre, Javier Ibañez-Guzmán, Christian Laugier
}

\begin{abstract}
Navigating through a road intersection is a complex manoeuvre that requires understanding the spatio-temporal relationships that exist between vehicles. Situation understanding and prediction are therefore fundamental functions for any computer-controlled safety or navigation system applied to road intersections. To interpret the situation at an intersection it is necessary to infer the intended manoeuvre of the relevant vehicles. Conventional approaches to manoeuvre prediction rely mainly on vehicle kinematics and dynamics. The contention of this paper is that contextual information in the form of topological and geometrical characteristics of the intersection can provide useful cues to understand the behaviour of a vehicle. We describe a probabilistic framework that extracts information from a digital map and uses it along with vehicle state information to estimate a driver's intended manoeuvre. The proposed approach is applicable to different types of intersections and handles uncertainty on the input information. We evaluate the performance of our approach on several real life scenarios using data recorded from real traffic.
\end{abstract}

\section{INTRODUCTION}

Intersections are among the most complex environments encountered in road networks. The large number of geometrical configurations, signalisation, traffic rules, and vehicle interactions results in many different possible scenarios. Accurate situation assessment by human drivers or software is difficult yet fundamental for the safe traversal of intersections. Difficult because of the large number of potential scenarios. Fundamental as misinterpretations could lead to hazardous situations resulting in accidents. Statistics show that in Europe (2004) $43 \%$ of road accidents resulting in injuries occurred at intersections [14], hence the interest by vehicle OEMs to develop Advanced Driver Assistance Systems (ADAS) to improve safety in these areas. Recent research endeavours on this topic include [11], [15]. The ability for a system to understand what is happening at an intersection and then act accordingly is of great importance if vehicles are to move autonomously, as was demonstrated at the DARPA Urban Challenge [4]. In particular, predicting the manoeuvre of vehicles is a crucial task since it helps identifying potential future collisions. Knowledge about drivers' manoeuvre intentions therefore impacts the ability of the system to provide relevant situation awareness and decision support at intersections.

The key issue for manoeuvre prediction is to be able to infer driver intent from incomplete models and uncertain data.

S. Lefèvre and J. Ibañez-Guzmán are with Renault S.A., 1 av. du Golf, 78288 Guyancourt, France, \{stephanie.lefevre, javier.ibanezguzman\}@renault.fr

S. Lefèvre and C. Laugier are with INRIA Grenoble Rhône-Alpes, 655 av. de l'Europe - Montbonnot, 38334 Saint Ismier Cedex, France, \{stephanie.lefevre, christian.laugier\}@inrialpes.fr
Incompleteness is a consequence of the fact that the underlying physics of the situation are so complex that it is not possible to design a model of the environment that can interpret a situation with no ambiguity from the available data. Uncertainty arises from the inability of sensors to measure perfectly the true state of the environment.

Conventionally in the literature the prediction of a vehicle's manoeuvre relies mainly on the interpretation of vehicle kinematics and dynamics. Map information is occasionally used, but only as constraints on the reachable positions. In this work the claim is that the contextual information contained in the map of the road network (i.e. spatial description of the intersection) is an important indication to interpret and predict the behaviour of a vehicle. This paper describes a probabilistic approach to the problem of driver intention estimation at intersections that centres on the use of map-based contextual information and on the handling of uncertainties. The proposed system exploits information about the layout of the intersection contained in the map together with observations on the vehicle. The uncertainty associated with the input information is taken into account.

The remainder of the paper is organised as follows. Section II gives an overview of related work. We describe the Bayesian Network proposed to model a vehicle negotiating an intersection in Section III, and we outline our approach to handle uncertainty on evidence in Section IV. In the last section we describe the experimental evaluation method and comment the results obtained by our approach.

\section{RELATED WORK}

This section gives an overview of approaches in the literature that have been applied to the manoeuvre prediction problem. So far, the idea of extracting useful information from a digital map to improve situation understanding has not been extensively explored, and very little effort has been made to take into account uncertainty on lane assignment. The approaches to manoeuvre prediction can be classified into two main groups: motion-based approaches and behaviour-based approaches.

Motion-based approaches use kinematic and/or dynamic models to describe a vehicle's motion and try to predict its state evolution (e.g. position, speed and orientation) over time when a control input (e.g. acceleration) is applied. The authors of [5] propose to rely on an occupancy grid and a Bayesian Occupancy Filter (BOF) [3] for estimating and predicting a vehicle's position. They restrict in a probabilistic way the 
reachable positions to the cells of the grid that are part of the road network.

Behaviour-based approaches exploit the fact that in a given context, vehicles often exhibit typical motion patterns. If one can identify the behaviour that a vehicle is following, the motion pattern can be used to predict motion. Behaviour-based approaches can be further classified into discriminative and generative methods.

Discriminative approaches can be used to classify driver intention without modelling explicitly the underlying distribution of variables and features. For trajectory classification, a popular method is to cluster trajectories into prototype trajectories [2]. At a higher level, the work described in [1] uses Support Vector Machines (SVMs) coupled with Bayesian filtering to infer agent intentions at intersections (harmless v.s. dangerous agent classification). The challenge with this kind of approaches is to be able to build a generic system that is applicable to every intersection. Because the geometry of intersections varies significantly, it is difficult to select a set of meaningful generic features to work with, and the training process requires a large amount of data from many different types of intersections.

Generative methods explicitly model the process that leads to assessing driver intention. A popular approach is to decompose complex manoeuvres into sequences of simpler events. Hidden Markov Models (HMMs) are a powerful tool to do this and are used in several works [9], [8]. Typical motion patterns can be learned and then used to predict motion on the basis of sensor data using the Growing HMM (GHMM) paradigm [16]. The author of [13] proposes to apply Hierarchical HMMs (HHMMs) to manoeuvre recognition and to use Gaussian Processes to represent the uncertainty on the realisation of a manoeuvre. Fuzzy logic is used in combination with a Probabilistic Finite State Machine (PFSM) in [6], while the authors of [7] propose to model driver behaviour using a PFSM where the transition probabilities are set dynamically using the output of a continuous vehicle state tracker.

These approaches either assume lane-level positioning or can only be applied to one specific type of intersection or do not use information about the layout of the intersection. As an alternative, the next two sections describes a behaviourbased generative approach that we propose as a generic solution to the problem of predicting a vehicle's manoeuvre at an intersection. The focus is on exploiting the contextual information provided by the digital map, and on the handling of uncertainties.

\section{A CONTEXT-BASEd BAyesian Network to MOdel INTERSECTION NEGOTIATION}

The first part of this section describes the information that is available for our system to infer a driver's intended manoeuvre. In the second part the Bayesian Network (BN) that was designed to model a vehicle negotiating an intersection is introduced.

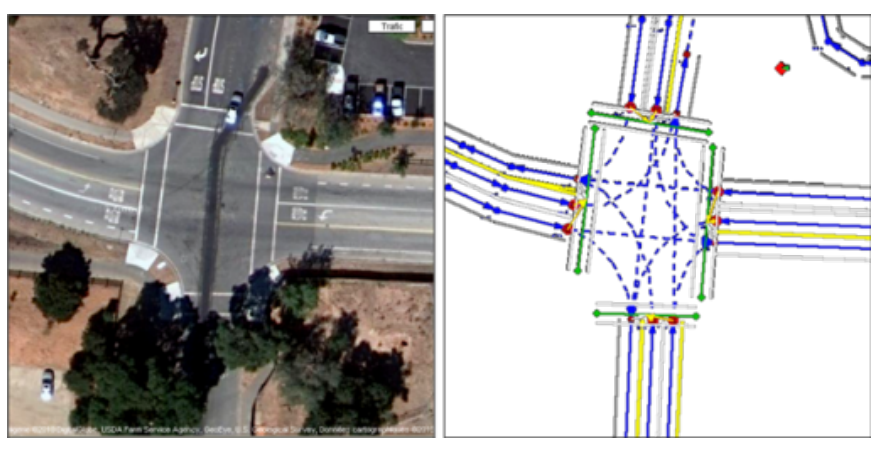

Figure 1. This intersection (satellite imagery on the left, RNDF representation on the right) has 4 roads, 8 entrance lanes, 4 exit lanes and 12 crossing connections. The blue arrows represent the centre of lanes and the dotted arrows inside the intersection area are the exemplar paths generated for each crossing connection. The green lines show the position of crosswalks. The red dots are the delimiters between the inside and the outside of the intersection.

\section{A. Input information}

1) Digital map of the road network: The map of the road network used in this work is in the Route Network Definition File (RNDF) format, a representation that was defined for the DARPA Urban Challenge [4]. Intersections are represented using topological and geometrical information: they consist of a list of roads, lanes, and authorised crossing connections. Adjacent lanes are part of the same road. In addition to this information, exemplar paths are generated. They correspond to the generic possible paths that a vehicle can take inside the intersection; a path is generated for each authorised crossing connection. This representation is illustrated in Fig. 1. Our map was built manually using a tool to annotate satellite images, and the resulting accuracy is approximately $0.5 \mathrm{~m}$.

2) Vehicle state information: The observations consist of the successive turn signal states, positions and orientations of the vehicle. There is no constraint on how this information should be obtained; it can be either from proprioceptive sensors of the ego-vehicle (if one is interested in predicting the ego-vehicle's manoeuvre), from exteroceptive sensors or from V2X communication (if one is interested in predicting the manoeuvre of other vehicles).

\section{B. Model description}

We model a vehicle approaching and traversing an intersection by the Bayesian Network represented in Fig. 2. The variables are defined below:

- $R \in\left\{r_{i}\right\}_{1}^{N_{R}}$ : the road through which the vehicle reaches the intersection

- $L \in\left\{l_{i}\right\}_{1}^{N_{L}}$ : the entrance lane through which the vehicle reaches the intersection

- $T \in\{$ left,right, none $\}$ : the turn signal that is on

- $P \in\left\{p_{i}\right\}_{1}^{N_{P}}$ : the exemplar path that the vehicle follows in the intersection

- $M \in\left\{m_{i}\right\}_{1}^{N_{M}}$ : the exit lane through which the driver intends to exit the intersection, i.e. the intended manoeuvre

The edges of the $\mathrm{BN}$ represent the causal dependencies between the variables. They can be interpreted as follows. When 


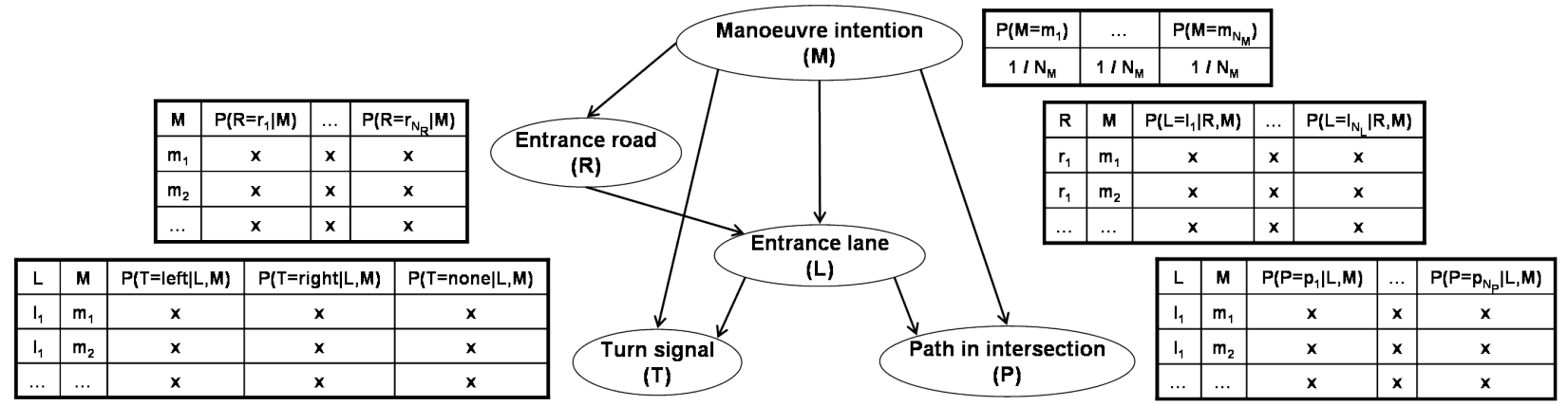

Figure 2. Bayesian Network model of a vehicle negotiating an intersection. The values $\mathrm{x}$ in the CPTs are calculated as described in Section III-B.

a driver approaches an intersection he intends to perform a specific manoeuvre (i.e. to reach a specific exit lane). To reach the intersection he will select an entrance road based on the intended manoeuvre, and an entrance lane based on the entrance road and the intended manoeuvre. He will also decide whether to put on a turn signal or not depending on the entrance lane and the intended manoeuvre. Once inside the intersection he will follow a path that is in accordance with the entrance lane and the intended manoeuvre.

The prior distribution on $M$ (i.e. $P(M)$ ) is set as uniform over all the possible exit lanes since there is no prior knowledge on the driver's intent. The values in the conditional probability tables (CPTs) are set by a rule-based algorithm that automatically computes them from the topological and geometrical characteristics of the intersection. This allows the system to be generic to any type and shape of intersection. The parameters that are taken into account by the rule-based algorithm are described in what follows.

1) $P\left(r_{i} \mid m_{i}\right)$ is a function of:

- whether or not entrance road $r_{i}$ has a lane that leads to exit lane $m_{i}$

- the number of roads that have a lane that leads to exit lane $m_{i}$

- a constant variable $\alpha$ that represents the probability that the driver will do a forbidden manoeuvre (in terms of allowed 'entrance road - exit lane' pairs)

2) $P\left(l_{i} \mid r_{i}, m_{i}\right)$ is a function of:

- whether or not entrance lane $l_{i}$ belongs to entrance road $r_{i}$

- whether or not entrance lane $l_{i}$ leads to exit lane $m_{i}$

- the number of lanes in entrance road $r_{i}$ that lead to exit lane $m_{i}$

- a constant variable $\beta$ that represents the probability that the driver will do a forbidden manoeuvre (in terms of allowed 'entrance road - entrance lane - exit lane' triplets)

3) $P\left(t_{i} \mid l_{i}, m_{i}\right)$ is a function of:

- whether or not exit lane $m_{i}$ is reachable from entrance lane $l_{i}$

- whether or not there exists an entrance lane that 1) leads to exit lane $m_{i}$ and 2) is different from entrance lane $l_{i}$ but belongs to the same road. If it is the case, $\mathrm{P}\left(t_{i} \mid l_{i}\right.$, $m_{i}$ ) will be a function of the position of that entrance lane with respect to entrance lane $l_{i}$ (left or right). This accounts for the fact that a vehicle driving on the left lane that puts its right turn signal on probably intends to reach an exit lane that is reachable from the right lane only.

- the angle formed by entrance lane $l_{i}$ and exit lane $m_{i}$

- whether or not exit lane $m_{i}$ is an extreme exit lane (extreme left or right) with respect to entrance lane $l_{i}$

- whether or not there exists exit lanes that are located on the same side (left or right) as exit lane $m_{i}$ with respect to entrance lane $l_{i}$

4) $P\left(p_{i} \mid l_{i}, m_{i}\right)$ is a function of:

- whether or not path $p_{i}$ originates from entrance lane $l_{i}$

- whether or not path $p_{i}$ leads to exit lane $m_{i}$

- the distance between the current position of the vehicle and entrance lane $l_{i}$. The idea is that when a vehicle is beginning its manoeuvre inside the intersection, it is likely to be on a path that originates from entrance lane $l_{i}$.

- the distance between the current position of the vehicle and exit lane $m_{i}$. The idea is that when a vehicle is finishing its manoeuvre inside the intersection, it is likely to be on a path that leads to exit lane $m_{i}$.

- the angle between exit lane $m_{i}$ and the tangent to path $p_{i}$ at the current best matched position of the vehicle on path $p_{i}$. This angle indicates where the vehicle might go if it follows path $p_{i}$ only temporarily.

In this work $\alpha$ and $\beta$ are arbitrarily set but in the future they should be learned from data.

\section{INTEGRATION OF UNCERTAIN EVIDENCE AND INFERENCE}

\section{A. Why the need for uncertain evidence?}

Using the model described in Section III-B, we wish to infer a discrete probability distribution on the intended manoeuvre $(M)$ from observations on the turn signal state $(T)$, the entrance lane taken by the vehicle $(L)$ and the path taken by the vehicle $(P)$. If there was no uncertainty on the values of $T, L$ and $P$ the inference equation to estimate $M$ would be $P(M \mid L, T$, $P$ ). However, performing lane assignment (and thus knowing with certainty the value of $L$ and $P$ ) requires that the error on positioning, object detection and the digital map be less than 
$0.3 \mathrm{~m} \mathrm{[11].} \mathrm{This} \mathrm{level} \mathrm{of} \mathrm{accuracy} \mathrm{for} \mathrm{positioning} \mathrm{is} \mathrm{not} \mathrm{a}$ reality yet for passenger cars. Therefore hard lane assignment is not feasible; it is necessary that the system handles uncertain evidence on $L$ and $P$. The system should also handle uncertain information on $T$ since in some cases the detection of turn signal state will be uncertain (e.g. if the turn signals of other vehicles are detected from camera images in a probabilistic manner).

In this work, evidence for the BN takes the form of discrete probability distributions on $T, L$ and $P$, denoted by $\lambda_{T}, \lambda_{L}$ and $\lambda_{P}$ respectively. The manner in which these probability distributions are generated is explained in the next paragraphs, as well as the method used to incorporate this uncertain information into the $\mathrm{BN}$.

\section{B. Generating uncertain evidence}

The input information (see Section III-A) needs to be converted to discrete probability distributions $\lambda_{T}, \lambda_{L}$ and $\lambda_{P}$.

$\lambda_{T}$ is defined as $\lambda_{T}=\left(\lambda_{\text {left }}, \lambda_{\text {right }}, \lambda_{\text {none }}\right)$, where $\lambda_{\text {left }}=$ $P(T=l e f t)$ (and similarly for right and none). It is obtained directly from the input information.

$\lambda_{L}$ is defined as $\lambda_{L}=\left(\lambda_{l_{1}}, \ldots, \lambda_{l_{N_{L}}}\right)$, where $\lambda_{l_{i}}=P(L=$ $\left.l_{i}\right)$. We represent a lane as a bivariate normal distribution on successive (position, orientation) states, therefore the likelihood $\lambda_{l_{i}}^{\prime}$ of a vehicle being on lane $l_{i}$ is calculated as

$$
\lambda_{l_{i}}^{\prime}=\exp \left(-\frac{1}{2}\left(\frac{\delta_{i}^{2}}{\sigma_{\delta}^{2}}+\frac{\theta_{i}^{2}}{\sigma_{\theta}^{2}}\right)\right),
$$

where $\delta_{i}$ is the distance between the vehicle's position and its orthogonal projection on lane $l_{i}, \theta_{i}$ is the angle between the vehicle's orientation and the orientation of lane $l_{i}, \sigma_{\delta}$ (resp. $\sigma_{\theta}$ ) is the standard deviation set for the distance (resp. the angle). In this work $\sigma_{\delta}$ and $\sigma_{\theta}$ are fixed, but a method to take into account the covariance matrices of the vehicle state and of the map can be found in [12]. Then the probability distribution $\lambda_{L}$ is computed by normalising the likelihoods $\lambda_{l_{i}}^{\prime}$ :

$$
\lambda_{l_{i}}=\frac{\lambda_{l_{i}}^{\prime}}{\sum_{i=1}^{N_{L}} \lambda_{l_{i}}^{\prime}} .
$$

$\lambda_{P}$ is defined as $\lambda_{P}=\left(\lambda_{p_{1}}, \ldots, \lambda_{p_{N_{P}}}\right)$, where $\lambda_{p_{i}}=P(P=$ $\left.p_{i}\right)$. The calculation is similar to the calculation of $\lambda_{L}$.

\section{Integrating uncertain evidence in the $B N$}

1) Addition of virtual nodes to the $B N$ : One advantage of using probability distributions directly as evidence for a $\mathrm{BN}$ is that it makes the handling of uncertainties very flexible. Instead of trying to represent explicitly in the BN the underlying variables that introduce uncertainties, the probability distributions over the uncertain variables are computed independently of the $\mathrm{BN}$.

The concept of using a discrete probability distribution as evidence in a BN is sometimes referred to as virtual evidence and was introduced as a generalisation of the standard hard evidence by Pearl [10]. In what follows we describe the process of incorporating in our BN uncertain evidence on the

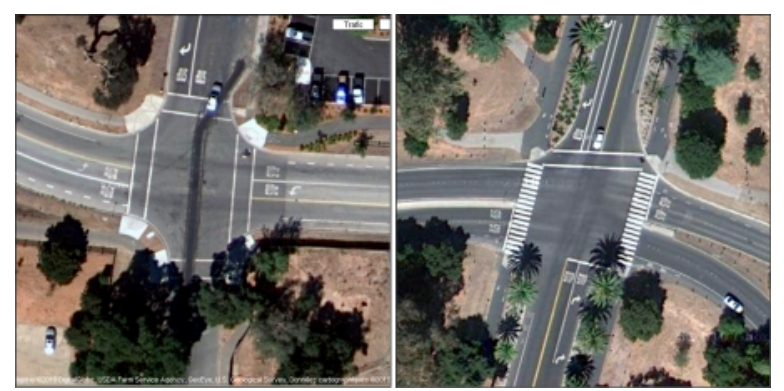

Figure 3. The four-way stop intersections at which experimental evaluation was performed.

value of $T$. A node $V_{T}$ is added as a child of node $T$. $V_{T}$ is called a virtual node and corresponds to a binary variable always observed to be 1 . The conditional probability table $P\left(V_{T} \mid T\right)$ is set dynamically using $\lambda_{T}$ as follows:

$$
\begin{cases}P\left(V_{T}=1 \mid T=\text { left }\right) & =\lambda_{\text {left }} \\ P\left(V_{T}=1 \mid T=\text { right }\right) & =\lambda_{\text {right }} \\ P\left(V_{T}=1 \mid T=\text { none }\right) & =\lambda_{\text {none }}\end{cases}
$$

The same process is applied for integrating in the BN uncertain evidence on $L$ and $P$ : virtual nodes $V_{L}$ and $V_{P}$ are added as children of $L$ an $P$ respectively, and the conditional probability tables $P\left(V_{L} \mid L\right)$ and $P\left(V_{P} \mid P\right)$ are set dynamically using $\lambda_{L}$ and $\lambda_{P}$ respectively.

2) Inference equation with uncertain evidence: The joint probability function of the BN of Fig. 2 after the virtual nodes have been added is:

$$
\begin{aligned}
P\left(M, R, L, T, P, V_{T}, V_{L}, V_{P}\right)= & P(M) \times P(R \mid M) \\
& \times P(T \mid M, L) \times P\left(V_{T} \mid T\right) \\
& \times P(L \mid M, R) \times P\left(V_{L} \mid L\right) \\
& \times P(P \mid M, L) \times P\left(V_{P} \mid P\right)
\end{aligned}
$$

The inference equation to estimate $M$ from uncertain evidence on $L, T$ and $P$ is $P\left(M \mid V_{T}=1, V_{L}=1, V_{P}=1\right)$. Its analytical form can be derived from Eq. 1; we then perform exact inference.

\section{EXPERIMENTAL EVALUATION}

\section{A. Evaluation data and scenarios}

The system is tested on 42 vehicle trajectories recorded from real traffic at two different intersections (see Fig. 3).

Evidence on $L$ and $P$ is obtained by labelling laser data (position and orientation of the vehicles) and applying the method described in Section IV-B.

Evidence on $T$ is automatically generated based on the scenario we wish to test the system on. We defined 4 scenarios; they are illustrated in Table I and use the following terminology:

- Consistent behaviour: the turn signal $T$ is consistent with the manoeuvre $M$ (e.g. no turn signal or left turn signal on for a left turn). 


\begin{tabular}{|c|c|c|}
\hline Id & Scenario description & Example \\
\hline 1 & $\begin{array}{c}\text { Consistent behaviour } \\
+ \\
\text { High confidence in turn signal } \\
\text { information }\end{array}$ & \\
\hline 2 & $\begin{array}{c}\text { Consistent behaviour } \\
+ \\
\text { Low confidence in turn signal } \\
\text { information }\end{array}$ & \\
\hline 3 & $\begin{array}{c}\text { Inconsistent behaviour } \\
+ \\
\text { High confidence in turn signal } \\
\text { information }\end{array}$ & \\
\hline 4 & $\begin{array}{c}\text { Inconsistent behaviour } \\
+ \\
\text { Low confidence in turn signal } \\
\text { information }\end{array}$ & \\
\hline
\end{tabular}

Table I

DEFINITION OF THE EVALUATION SCENARIOS

- Inconsistent behaviour: the turn signal $T$ is not consistent with the manoeuvre $M$ (e.g. right turn signal on for a left turn).

- High confidence in turn signal information: there is little uncertainty on the turn signal state.

In this situation the probability distribution on $T$ will be set to $\lambda_{T}=(0.9,0.05,0.05)$ or $\lambda_{T}=(0.05,0.9,0.05)$ or $\lambda_{T}=(0.05,0.05,0.9)$.

- Low confidence in turn signal information: there is a high uncertainty on the turn signal state.

In this situation the probability distribution on $T$ will be set to $\lambda_{T}=(0.4,0.3,0.3)$ or $\lambda_{T}=(0.3,0.4,0.3)$ or $\lambda_{T}=(0.3,0.3,0.4)$.

The idea behind the definition of these scenarios is to test our system on a variety of real life situations. Drivers sometimes unintentionally misuse turn signals (thus the definition of consistent and inconsistent behaviours), and the confidence in the information on turn signal varies significantly depending on the way it was obtained (see Section III-A2).

\section{B. Evaluation method}

The performance is evaluated by measuring how early the system is capable of making a correct prediction about the manoeuvre of a vehicle in each of the scenarios. For a trajectory with ground truth $M=m_{k}$ (i.e. the driver actually exits the intersection through exit lane $m_{k}$ in the end), an individual prediction will be considered correct if

$$
\underset{i}{\operatorname{argmax}}\left(P\left(M=m_{i} \mid V_{T}, V_{L}, V_{P}\right)\right)=k .
$$

For each tested trajectory we determine the timestep $t_{c}$ such that all the subsequent predictions are correct and we define $\delta_{c} \in[0,50]$ as the distance in meters between the vehicle and the exit lane $m_{k}$ at timestep $t_{c}$. The upper bound for $\delta_{c}$ delimits the area where evaluation is performed at the intersection. In this work it is set to $50 \mathrm{~m}$, which corresponds to the distance until which we are able to label vehicles in our laser data.

In order to evaluate the performance of the system we consider the distribution of $\delta_{c}$ in each of the scenarios defined in Section V-A. This allows us to determine how the inconsistencies in a driver's behaviour and the confidence in the turn signal information impact the ability of the system to infer the driver's intention.

\section{Results and interpretation}

The system is tested on 42 trajectories for each scenario. The distributions of $\delta_{c}$ are plotted in Fig. 4 and can be interpreted as follows.

1) Scenario 1: In this scenarios $\delta_{c}=50$ for all the trajectories, which means that the system makes correct predictions for every trajectory at every timestep. This is not surprising since in this scenario strong cues are available to predict the manoeuvre of a vehicle (high confidence in the turn signal information) and these cues are never misleading (consistent behaviour).

2) Scenario 2: When the turn signals are used correctly by the drivers but the uncertainty on turn signal information is high, the system occasionally makes incorrect predictions over the course of a trajectory. In our dataset these incorrect predictions all happened in the following situation: a driver intends to make a left turn but starts steering left particularly late in the intersection. In this situation the system makes correct predictions until the vehicle reaches the centre of the intersection. Then during a few timesteps just before the driver starts steering left, the system interprets the absence of steering as an intention to go straight. As a result the system's prediction becomes incorrect for a brief period. This explains why $10<\delta_{c}<20$ for $5 \%$ of the trajectories.

These events, even if rare, uncover one limitation of our approach: our exemplar paths do not account for the fact that there are variations in the way people execute a manoeuvre in an intersection. In such situations, a reliable information on turn signal state compensates for the inaccuracy of exemplar paths (Scenario 1) but a high uncertainty on turn signal state sometimes does not (Scenario 2).

3) Scenario 3: When the confidence in the turn signal information is high and the driver misuses the turn signals, the system's predictions will be incorrect until there are enough clues (position, orientation of the vehicle) that contradict the turn signal clue. The distance $\delta_{c}$ will therefore vary depending on how early in the manoeuvre the different clues conflict with each other.

If at the beginning of the manoeuvre the clues are not conflicting (e.g. the vehicle has its left turn signal on and is located on a lane from which left turn manoeuvres are allowed), the misuse of the turn signal will becomes obvious only close the end of the manoeuvre, when the exit lanes indicated by the turn signal are no longer reachable. In our dataset $41 \%$ of the trajectories fit this case, i.e. the manoeuvre 

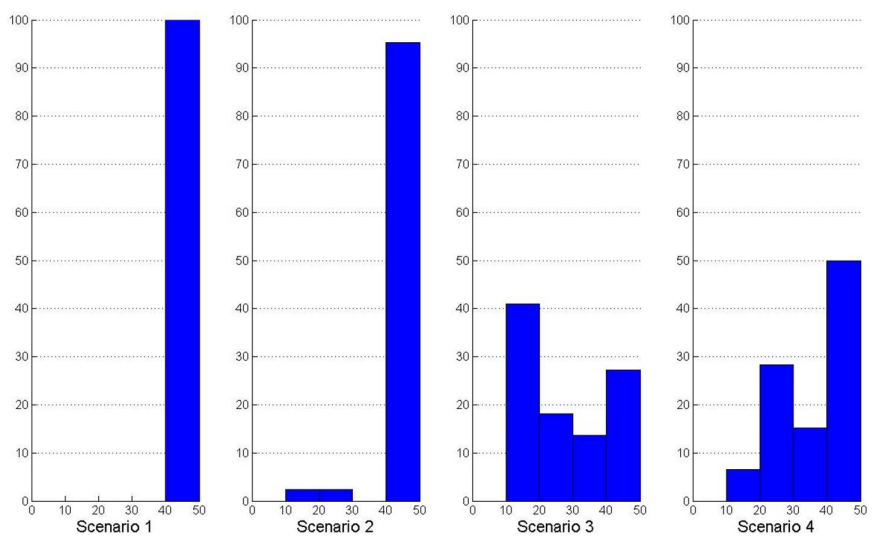

Figure 4. Histogram representation of the distribution of $\delta_{c} \in[0,50]$ for each of the scenarios defined in Section V-A.

is not identified correctly until the vehicle is located between 10 and $20 \mathrm{~m}$ from its exit lane.

If the clues are conflicting starting from the beginning of the manoeuvre (e.g. the vehicle has its left turn signal on and is located on a lane from which left turn manoeuvres are not allowed), the misuse of the turn signal will becomes obvious much earlier. $28 \%$ of the tested trajectories fit this case, i.e. the manoeuvre is identified correctly when the vehicle is more than $40 \mathrm{~m}$ away from its exit lane.

In all cases $\delta_{c}$ is higher than $10 \mathrm{~m}$.

4) Scenario 4: When the information on the turn signal state is misleading but uncertain, the system is able to make correct predictions much earlier (in comparison with Scenario 3): $\delta_{c}$ is less than $20 \mathrm{~m}$ in only $7 \%$ of the cases (against $41 \%$ in Scenario 3), and in $50 \%$ of the cases the system makes correct predictions when the vehicle is more than $40 \mathrm{~m}$ away from its exit lane (against $28 \%$ in Scenario 3 ).

The differences in the results between the two scenarios can be explained as follows. In Scenario 3 there is only one correct interpretation of the conflicting data, which is that the driver is misusing the turn signals. A driver misusing the turn signals is a rare event, it is therefore unlikely and the system will prefer another interpretation until late in the manoeuvre. In Scenario 4 there are two possible correct interpretations for the conflicting data, which are that the driver is misusing the turn signals or that the true turn signal state is not the one presented as the most likely by the input information. Since the confidence in the input information on turn signals is low, the system will favour this (correct) interpretation over the other ones early in the manoeuvre.

\section{CONCLUSIONS}

In this paper a novel approach for predicting the manoeuvre of a vehicle at an intersection was introduced. The proposed method extracts contextual information from a digital map and integrates it in a Bayesian Network that models a vehicle negotiating a road intersection. The system is generic to any intersection layout and is capable of handling uncertain information. The performance of the system was evaluated in four scenarios including cases where the driver misuses turn signals.

Future work will integrate vehicle dynamics to allow for more complex manoeuvre recognition. The variability on the execution of a manoeuvre in an intersection should be accounted for in the exemplar paths. The idea of using contextual information will be investigated further: priority rules will be taken into account to improve situation assessment at an intersection.

\section{ACKNOWLEDGEMENTS}

This work is the result of a collaboration with the Stanford Artificial Intelligence Lab. The authors would like to thank Prof. S. Thrun for accepting the first author as a visiting researcher in his group, as well as Dr. S. Kammel and M. Sokolsky for their support in integrating this work into the Stanford Driving Group framework.

The authors are grateful for helpful discussions with $\mathrm{M}$. Sokolsky, Dr. I. Paromtchik and Dr. M. Perrollaz

\section{REFERENCES}

[1] G. S. Aoude and J. P. How. Using support vector machines and bayesian filtering for classifying agent intentions at road intersections. Technical report, Aerospace Controls Laboratory, Department of Aeronautics and Astronautics, Massachusetts Institute of Technology, 2009. Report ACL09-02.

[2] S. Atev, G. Miller, and N. Papanikolopoulos. Clustering of vehicle trajectories. IEEE Transactions on Intelligent Transportation Systems, 11(3):647-657, 2010.

[3] C. Coué, C. Pradalier, C. Laugier, T. Fraichard, and P. Bessière. Bayesian occupancy filtering for multitarget tracking: an automotive application. International Journal of Robotics Research, 25(1):19-30, 2006.

[4] DARPA Urban Challenge. http://www.darpa.mil/grandchallenge.

[5] T. Gindele, S. Brechtel, J. Schröder, and R. Dillmann. Bayesian occupancy grid filter for dynamic environments using prior map knowledge. In Proc. IEEE Intelligent Vehicles Symposium, 2009.

[6] T. Hulnhagen, I. Dengler, A. Tamke, T. Dang, and G. Breuel. Maneuver recognition using probabilistic finite-state machines and fuzzy logic. In Proc. IEEE Intelligent Vehicles Symposium, pages 65-70, 2010.

[7] A. Kurt, J. L. Yester, Y. Mochizuki, and Ümit Özgüner. Hybrid-state driver/vehicle modelling, estimation and prediction. In Proc. IEEE Intelligent Transportation Systems Conference, pages 806-811, 2010.

[8] D. Meyer-Delius, C. Plagemann, and W. Burgard. Probabilistic situation recognition for vehicular traffic scenarios. In Proc. IEEE International Conference on Robotics and Automation, pages 4161-4166, 2009.

[9] N. Oliver and A. P. Pentland. Graphical models for driver behavior recognition in a SmartCar. In Proc. IEEE Intelligent Vehicles Symposium, pages 7-12, 2000.

[10] J. Pearl. Probabilistic reasoning in intelligent systems: networks of plausible inference. Morgan Kaufmann, 1988.

[11] PReVENT project - INTERSAFE subproject. Final report (d40.75), February 2007.

[12] R. Schubert and G. Wanielik. A unified Bayesian approach for tracking and situation assessment. In Proc. IEEE Intelligent Vehicles Symposium, pages $738-745,2010$.

[13] C. Tay. Analysis of dynamic scenes: application to driving assistance. PhD thesis, Institut National Polytechnique de Grenoble, France, 2009.

[14] TRACE project. http://www.trace-project.org/.

[15] U.S. DOT's CICAS initiative. http://www.its.dot.gov/cicas/cicas.

[16] D. Vasquez, T. Fraichard, and C. Laugier. Growing Hidden Markov Models: an incremental tool for learning and predicting human and vehicle motion. The International Journal of Robotics Research, 28(1112):1486-1506, 2009. 